\title{
Tumor Microenvironment and RIG-I Signaling Molecules in Epstein Barr Virus-Positive and -Negative Classical Hodgkin Lymphoma of the Elderly
}

\author{
Tsugumi Satoh, ${ }^{1)}$ Ryuichi Wada, ${ }^{1)}$ Nobuhisa Yajima, ${ }^{1)}$ Tadaatsu Imaizumi, ${ }^{2)}$ and Soroku Yagihashi ${ }^{1)}$
}

Classical Hodgkin lymphoma (CHL) is a B-cell neoplasm characterized by Hodgkin and Reed-Sternberg (HRS) cells. Its prevalence exhibits a bimodal pattern of peaking in young adults and the elderly. There is an association with Epstein-Barr virus (EBV) infection in about $50 \%$ of cases of CHL of the elderly, and the outcome of these patients is unfavorable. It is not well known how the latent infection of EBV is involved in the pathophysiology of CHL of the elderly. To address this issue, we examined the tumor microenvironment (TME) and the expression of molecules related to EBV infection in HRS cells in 10 EBV-positive CHL and 7 EBV-negative CHL patients older than 50 years. In EBV-positive CHL, we found an increased population of $\mathrm{FOXP}^{+}$cells, while that of granzyme $\mathrm{B}^{+}$cells was reduced, compared with those in EBV-negative CHL. The expression of inhibitory chemokine CCL20 was increased in EBV-positive HRS cells compared with that in EBV-negative HRS cells. In addition, despite increased expression of a pattern recognition receptor, RIG-I, in intracellular innate immunity, there was no evidence of interferon regulatory factor 3 activation or interferon- $\beta$ induction in EBV-positive HRS cells in CHL of the elderly. The disease recurred frequently (50\%) in EBV-positive CHL. The current study thus suggests the possibility that the latent infection of EBV alters the expression of chemokines and the innate immunity response in HRS cells and modulates TME to an immunosuppressive state, which may account for the unfavorable disease course in CHL of the elderly. $\int J$ Clin Exp Hematop 54(1): 75-84, 2014]

Keywords: classical Hodgkin lymphoma, Epstein-Barr virus, tumor microenvironment, RIG-I, CCL20

\section{INTRODUCTION}

Classical Hodgkin lymphoma (CHL) is a B-cell-derived malignant lymphoma that shows a bimodal age distribution with peaks in young adults and the elderly. ${ }^{1}$ The histology of CHL is characterized by the appearance of Hodgkin and Reed-Sternberg (HRS) cells, which constitute approximately $1 \%$ of the cells in the tumor. ${ }^{2}$ HRS cells are found in a tumor microenvironment (TME) that consists of lymphocytes, neutrophils, eosinophils, mast cells, macrophages and fibroblasts. Survival of HRS cells is dependent on the cytokine and chemokine signals and cell-to-cell contact in the TME. ${ }^{3}$ HRS cells can evade immune attack from the infiltrated cells in the

\footnotetext{
Received : October 24, 2013

Revised : November 14, 2013

Accepted : December 18, 2013

${ }^{1)}$ Department of Pathology and Molecular Medicine, Hirosaki University Graduate School of Medicine, Hirosaki, Japan

${ }^{2)}$ Department of Vascular Biology, Institute of Brain Science, Hirosaki University Graduate School of Medicine, Hirosaki, Japan

Corresponding author: Ryuichi Wada, M.D., Ph.D., Department of Diagnostic Pathology, Nippon Medical School Hospital, 1-1-5 Sendagi, Bunkyo-ku, Tokyo 1138602, Japan

E-mail: w-ryuichi@nms.ac.jp
}

TME. The interaction between HRS cells and TME is considered to affect the clinical behavior of CHL.

There is an association with latent infection of Epstein Barr virus (EBV) in approximately $50 \%$ of cases of CHL. ${ }^{4}$ The EBV infection in CHL varies with age, and it is more common in patients older than 50 years. The infection often results in treatment failure and a poor prognosis. ${ }^{5-7}$ It is not known, however, how EBV is involved in the pathogenesis and progression of CHL.

In the latent infection of EBV, EBV-associated molecules such as Epstein Barr nuclear antigens (EBNAs), latent membrane proteins (LMPs), EBV-encoded RNA (EBER) and BamHI-A rightward transcripts are expressed. ${ }^{8}$ These molecules induce constitutive activation of $\mathrm{NF}-\varkappa \mathrm{B}$, which stimulates the survival signals and induces the expression of cytokines and chemokines, essential to the interaction with the cells in TME. Recently, it was shown that EBV-associated molecules may also trigger intracellular innate immunity. ${ }^{9}$ It was shown that EBER interacted with retinoic acid-inducible gene-I (RIG-I), a pattern recognition receptor, that in turn activated innate immunity in a Burkitt lymphoma (BL) cell line. ${ }^{10,11}$ At present, the alteration of intracellular RIG-I signaling in HRS cells of EBV-positive CHL is not known. 
The current study investigated the cellular composition of TME and the intracellular molecular alterations induced by EBV infection in CHL patients older than 50 years. We consider that an understanding of the molecular alterations would help to develop a new treatment strategy for EBVpositive CHL.

\section{PATIENTS AND METHODS}

\section{Patients}

The cases of CHL were obtained from the archives of pathology files of the Department of Pathology and Molecular Medicine, Hirosaki University. From 1995 to 2012, a total of 32 cases of CHL were procured. The age distribution was bimodal. The first peak was in the teens and the second peak was in the $60 \mathrm{~s}$. In the current study, 17 cases older than 50 years were used (Table 1). Hematoxylin and eosin sections of the cases were reviewed by TS, RW and NY, and the histological diagnosis was made in accordance with the criteria of the World Health Organization 2008. ${ }^{12}$ The presence of EBV was determined by in situ hybridization of EBER and immunostaining of LMP1.

\section{Diagnostic immunostaining}

Immunostaining with antibodies against CD30, CD15 and LMP1 (Dako Japan, Inc., Tokyo, Japan) was carried out with Ventana GX System (Roche Diagnostics, K.K., Tokyo, Japan). The peroxidase activity was visualized with diamino- benzidine, and the sections were counterstained with hematoxylin.

\section{In situ hybridization}

Detection of EBER was carried out with Ventana HX Discovery System (Roche Diagnostics, K.K.), using the mixture of anti-sense probes of digoxigenin-labeled synthesized RNA 5'-GGU UUU GCU AGG GAG GAG AC-3' and 5'UGA GGA CGG UGU CUG UGG UU-3.' As sense probes, synthesized RNA complementary to the anti-sense probes was used. After deparaffinization, the sections were treated with CC2 buffer and protease 2. Then, they were incubated with the probes at $70^{\circ} \mathrm{C}$ for $6 \mathrm{hr}$. After stringent washing with $0.1 \mathrm{XSSC}$ at $65^{\circ} \mathrm{C}$ for $6 \mathrm{~min}$, the sections were incubated with alkaline phosphatase (ALP)-labeled anti-digoxigenin antibody, and ALP was visualized using BlueMap Kit (Roche Diagnostics, K.K.). The sections were then counterstained with nuclear fast red.

\section{Morphometric analysis of cellular composition of TME}

The cellular composition of TME was evaluated by morphometry on the sections subjected to triple immunostaining of FOXP3, granzyme B (GrB) and CD30. Briefly, 3.5$\mu \mathrm{m}$-thick sections were deparaffinized, immersed in Tris (pH9.0)/EDTA buffer at $125^{\circ} \mathrm{C}$ for $5 \mathrm{~min}$, treated with Background Sniper (Biocare Medical, Concord, CA, USA) for $10 \mathrm{~min}$ and then incubated with the primary antibodies against $\mathrm{GrB}$ (1:500, Dako Japan, Inc.) at $37^{\circ} \mathrm{C}$ for $60 \mathrm{~min}$.

Table 1. Clinicopathological features of the cases

\begin{tabular}{|c|c|c|c|c|c|c|c|c|}
\hline No. & Age & Sex & Location & Subtype & CD30 & CD15 & EBER & LMP1 \\
\hline \multicolumn{9}{|c|}{ EBV-positive } \\
\hline 1 & 50 & M & Inguen & $\mathrm{MC}$ & + & + & + & + \\
\hline 2 & 53 & M & Cervix & NS & + & + & + & + \\
\hline 3 & 59 & M & Axilla & NS & + & - & + & + \\
\hline 4 & 63 & M & Cervix & $\mathrm{MC}$ & + & + & + & + \\
\hline 5 & 65 & M & Mediastinum & $\mathrm{MC}$ & + & + & + & + \\
\hline 6 & 68 & $\mathrm{~F}$ & Supraclavicule & NS & + & + & + & + \\
\hline 7 & 68 & M & Axilla & $\mathrm{MC}$ & + & + & + & + \\
\hline 8 & 68 & M & Supraclavicule & LR & + & - & + & + \\
\hline 9 & 84 & $\mathrm{~F}$ & Cervix & $\mathrm{MC}$ & + & - & + & + \\
\hline 10 & 86 & M & Inguen & $\mathrm{MC}$ & + & - & + & + \\
\hline \multicolumn{9}{|c|}{ EBV-negative } \\
\hline 11 & 53 & M & Cervix & NS & + & + & - & - \\
\hline 12 & 55 & M & Cervix & NS & + & - & - & - \\
\hline 13 & 58 & M & Inguen & LR & + & - & - & - \\
\hline 14 & 59 & $\mathrm{~F}$ & Cervix & MC & + & + & - & - \\
\hline 15 & 73 & M & Cervix & $\mathrm{MC}$ & + & + & - & - \\
\hline 16 & 74 & M & Inguen & NS & + & + & - & - \\
\hline 17 & 84 & $\mathrm{M}$ & Axilla & NS & + & - & - & - \\
\hline
\end{tabular}

EBV, Epstein-Barr virus; EBER, EBV encoded RNA; LMP-1, latent membrane protein 1; M, male; F, female; MC, mixed cellularity; NS, nodular sclerosis; LR, lymphocyte rich 
Following elimination of the endogenous peroxidase in $3 \%$ $\mathrm{H}_{2} \mathrm{O}_{2}$ in distilled water for $10 \mathrm{~min}$, sections were subjected to MACH3 Mouse HRP Polymer Detection (Biocare Medical). The reaction products were visualized as black with EnzMet (Nanoprobes, Inc., Yaphank, NY, USA). After the treatment with Denaturing Solution (Biocare Medical) for $5 \mathrm{~min}$ and with citrate buffer $(\mathrm{pH} 6.0)$ at $125^{\circ} \mathrm{C}$ for $3 \mathrm{~min}$, the sections were incubated with anti-FOXP3 antibody (1:300, Abcam, Tokyo, Japan) at $4^{\circ} \mathrm{C}$ overnight. They were processed with MACH3 Rabbit ALP Polymer Detection (Biocare Medical). Labeled ALP was colorized with Vulcan Fast Red Chromogen Kit 2 (Biocare Medical). The sections were treated again with Denaturing Solution (Biocare Medical) and citrate buffer at $125^{\circ} \mathrm{C}$ for $2 \mathrm{~min}$, and incubated with antiCD30 antibody (1:100, Dako Japan, Inc.) at $37^{\circ} \mathrm{C}$ for $75 \mathrm{~min}$. They were processed with ALP-labeled anti-mouse IgG antibody (Nichirei Biosciences, Inc., Tokyo, Japan), which was colorized with Ferrangi Blue Chromogen Kit 2 (Biocare Medical), followed by light counterstaining with hematoxylin.

For the morphometric analysis, 10 images of the immunostained sections with FOXP3, GrB and CD30, representative of a lesion containing HRS cells, were taken at a magnification of $x 400\left(1\right.$ image $\left.=0.033 \mathrm{~mm}^{2}\right)$ in each case. The numbers of $\mathrm{FOXP}^{+}$cells, $\mathrm{GrB}^{+}$cells and HRS cells in each image were counted, and the numbers of $\mathrm{FOXP}^{+}$and $\mathrm{GrB}^{+}$ cells were calculated as number of cells/HRS cell. From these values, the FOXP3/GrB ratio was obtained.

\section{Expression of CC chemokine ligand 20 (CCL20) and molecules of RIG-I signaling}

Immunostaining of RIG-I was carried out with Histofine SAB-PO (Nichirei Biosciences, Inc.). After blocking of endogenous peroxidase in $1 \% \mathrm{H}_{2} \mathrm{O}_{2}$ in methanol and treatment with normal rabbit serum, sections were incubated with antiRIG-I antibody (1:100, AbD Serotec, Oxford, UK) at $4^{\circ} \mathrm{C}$ overnight. They were then processed with Histofine, and labeled peroxidase was visualized with diaminobenzidine, followed by counterstaining with hematoxylin.

Expressions of CCL20, interferon regulatory factor 3 (IRF3), phosphorylated IRF3 (pIRF3) and interferon (IFN)- $\beta$ were examined by double immunostaining. After pretreatment in Tris (pH9.0)/EDTA buffer at $125^{\circ} \mathrm{C}$ for $3 \mathrm{~min}$, the sections were treated with Background Sniper (Biocare Medical) and incubated with the primary antibodies against CCL20 (1:500, Abcam), IRF3 (1:500, Santa Cruz Biotechnology, Inc., Dallas, TX, USA), pIRF3 (1:300, Thermo Scientific, Rockford, IL, USA) and IFN- $\beta$ (1:500, Novus Biologicals, LLC, Littleton, Co, USA) at $4^{\circ} \mathrm{C}$ overnight. After elimination of endogenous peroxidase, the sections were processed with MACH3 Rabbit HRP Polymer Detection (Biocare Medical) and visualized with EnzMet (Nanoprobes, Inc.). Following treatment with Denaturing
Solution (Biocare Medical) and citrate buffer, the sections were incubated with anti-CD30 antibody (Dako Japan, Inc.) at $37^{\circ} \mathrm{C}$ for $75 \mathrm{~min}$ and processed with MACH3 Mouse ALP Polymer Detection (Biocare Medical). The reaction products were visualized with Vulcan Fast Red Chromogen Kit 2 (Biocare Medical), followed by counterstaining with hematoxylin.

CCL20-positive HRS cells were identified by the cytoplasmic-positive reaction of CCL20 (black) and the membranous reaction of CD30 (red) under high magnification. CCL20-positive reactions in HRS cells were semi-quantified as follows: when double-positive cells were less than $10 \%$ of HRS cells, the score was 0 . When they exceeded $10 \%$, the score was further divided into 3 grades: score $1+$, weak but clearly positive, similar to the findings in inflammatory cells in TME; 2+, moderate with enhanced reaction compared with that of inflammatory cells; and $3+$, strongly positive reaction distinct from that of inflammatory cells.

\section{Statistical analysis}

Statistical analysis was performed with JMP10 (SAS Institute, Inc., Cary, NC, USA). Comparison of means in two groups was carried out by the Mann-Whitney method. The frequency distributions in two groups were compared by Chisquare test. The difference was considered significant when the $\mathrm{p}$ value was less than 0.05 .

\section{RESULTS}

\section{Clinicopathological features of the cases}

The ages of the patients ranged from 50 to 86 (median 65) years, and the male to female ratio was 14:3 (Table 1). All the specimens were from the lymph nodes, and most of them were biopsied from the neck and mediastinum.

Eight out of 17 cases were diagnosed as mixed cellularity subtype, and 7 cases were nodular sclerosis (NS). Two cases were lymphocyte-rich. CD30 was positive in HRS cells in all the cases (100\%) (Fig. 1), and CD15 was positive in 10 out of 17 cases $(59 \%)$. Ten out of 17 cases $(59 \%)$ were positive for EBER, and the positive signal was localized in the nuclei of the HRS cells (Fig. 1). LMP1 was also expressed in these 10 cases $(59 \%)$. The frequency of EBV positivity was $75 \%(6 / 8$ cases) in mixed cellularity subtype and $43 \%$ (3/7 cases) in nodular sclerosis subtype.

Follow-up data were available in 10 out of 17 patients. Among 6 cases of EBV-positive CHL that were followed up, recurrence was noted in 3 cases (50\%; Nos. 2, 4 and 5). Two patients are alive without disease (Nos. 6 and 9) and one died of pancreatic cancer (No. 1). Among 4 cases of EBVnegative $\mathrm{CHL}$ that were followed up, there was no recurrence $(0 \%)$ and only one died of progressive disease (No. 13). 
Three patients are alive (Nos. 11, 14 and 15)

\section{Cellular composition of TME}

The cellular composition of TME was examined by triple immunostaining of FOXP3 (red), GrB (black) and HRS cells (blue) (Fig. 2A, 2B). CD56-positive NK cells were scarcely observed in TME of either EBV-positive or -negative CHL (data not shown). $\mathrm{FOXP3}^{+}$and $\mathrm{GrB}^{+}$cells were distributed diffusely in TME in both EBV-positive and -negative CHL (Fig. 2A, 2B). In EBV-positive CHL, FOXP3 ${ }^{+}$cells appeared to surround HRS cells (Fig. 2A, arrowheads).

Morphometry revealed a significant increase in the proportion of $\mathrm{FOXP}^{+}$cells in EBV-positive CHL compared with

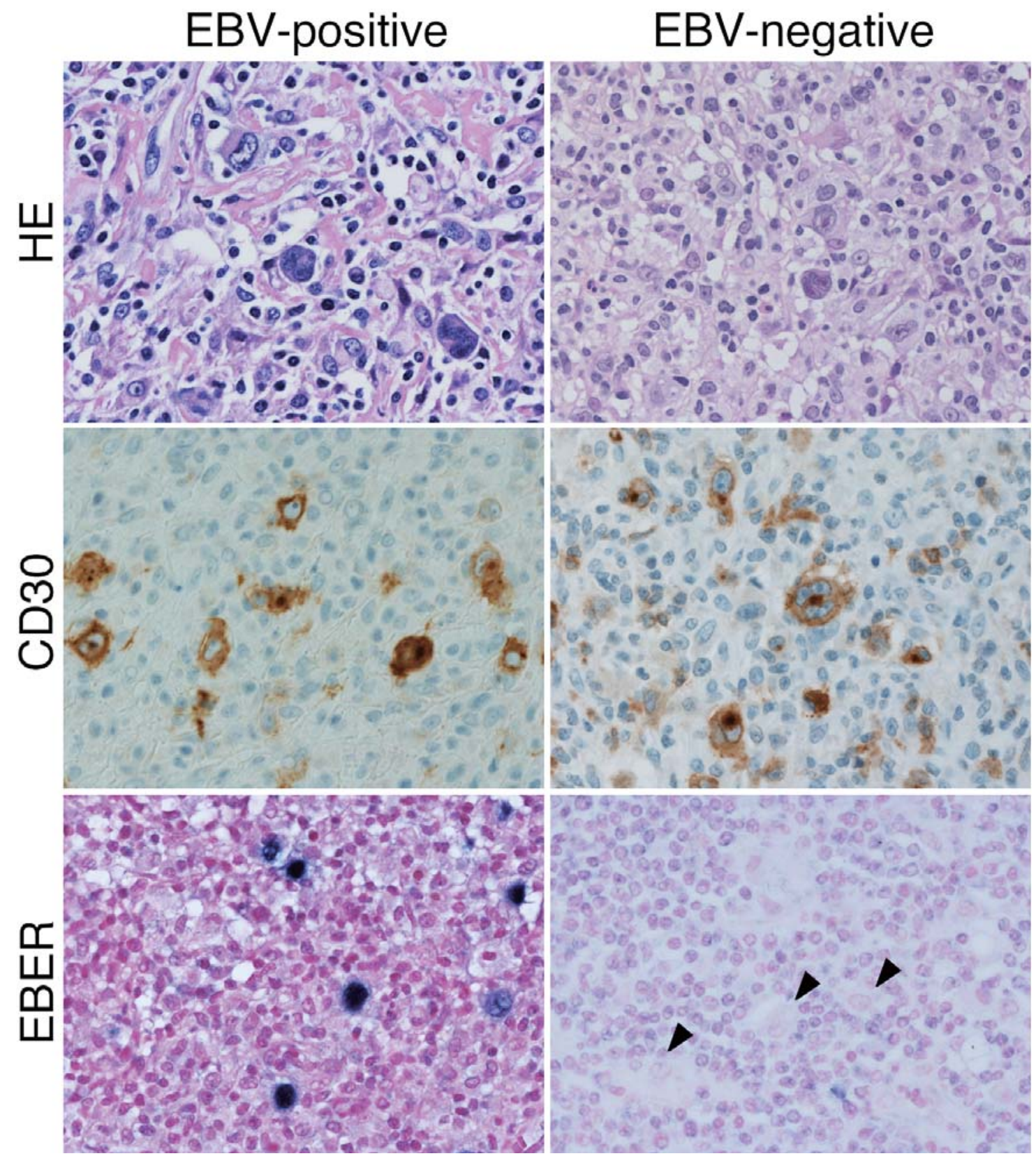

Fig. 1. Histology of classical Hodgkin lymphoma (CHL). Representative hematoxylin \& eosin staining (HE), immunostaining of CD30 and in situ hybridization of Epstein-Barr virus (EBV)-encoded RNA (EBER) of EBVpositive CHL (case 7) and EBV-negative CHL (case 16). Arrowheads indicate Hodgkin and Reed-Sternberg cells. 
that in EBV-negative CHL (Fig. 2C \& Table 2), and the $\mathrm{GrB}^{+}$ cells were significantly reduced in EBV-positive CHL (Fig. 2D \& Table 2). FOXP3/GrB ratio was therefore significantly higher in EBV-positive CHL than in EBV-negative CHL (Fig. 2E \& Table 2).

\section{Expression of CCL20}

CCL20 was expressed in HRS cells, adjacent small lymphocytes and neutrophils (Fig. 3). The intensity of CCL20 reactions in HRS cells was significantly enhanced in EBV- positive CHL compared with that in EBV-negative CHL (Tables $2 \& 3$ ).

\section{Expression of RIG-I signaling molecules}

RIG-I was expressed in all the cases of EBV-positive CHL (Fig. $3 \&$ Table 2). The positive reaction was noted in the cytoplasm of the HRS cells, and the intensity appeared to vary from cell to cell in each case. In EBV-negative CHL, the expression of RIG-I in HRS cells was not apparent.

IRF3 was expressed in HRS in both EBV-positive and
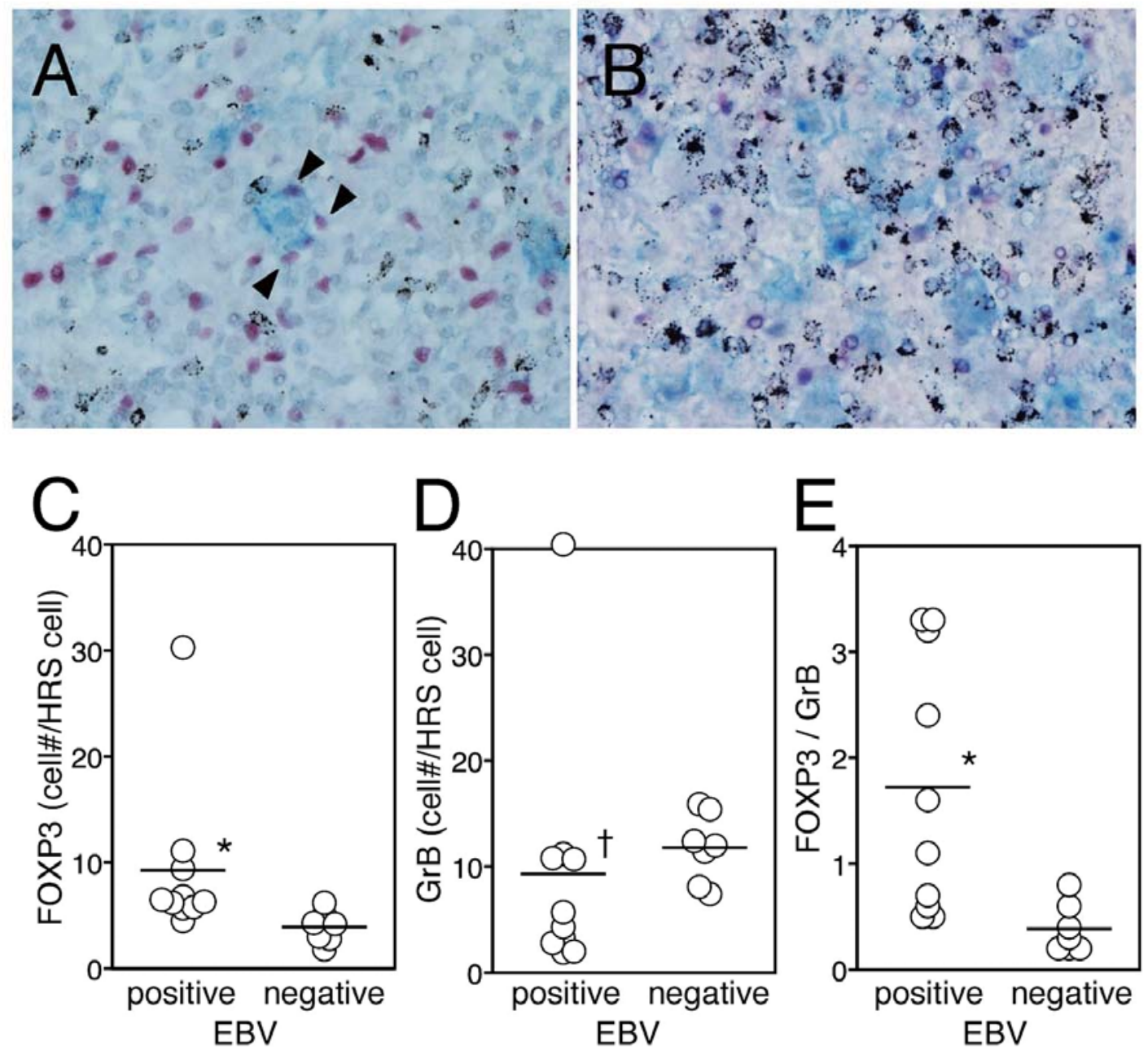

Fig. 2. Cellular composition of the tumor microenvironment in classical Hodgkin lymphoma (CHL). (2A \& $2 B$ ) The triple staining of FOXP3 (red), granzyme B (GrB, black) and CD30 (blue) in Epstein-Barr virus (EBV)-positive CHL (case 9) (2A) and EBV-negative CHL (case 16) (2B). $\mathrm{FOXP}^{+}$cells surrounding Hodgkin and Reed-Sternberg (HRS) cells are indicated with arrowheads. (2C) Compared with EBVnegative CHL, morphometry disclosed a significant increase in FOXP3 ${ }^{+}$cells in EBV-positive CHL $(*, p<$ 0.01). positive cells/HRS cell, cell\#/HRS cell. (2D) Granzyme B $(\mathrm{GrB})^{+}$cells were significantly reduced in EBV-positive CHL $(\dagger, \mathrm{p}<0.05)$. (2E) FOXP3/GrB ratio was significantly higher in EBV-positive CHL (*, $\mathrm{p}<0.01)$. 
Table 2. The cellular composition of tumor microenvironment and expression of molecules in classical Hodgkin lymphoma

\begin{tabular}{|c|c|c|c|c|c|c|c|c|}
\hline No. & FOXP3 & GrB & FOXP3/GrB & CCL20 & RIG-I & IRF3 & pIRF3 & IFN- $\beta$ \\
\hline \multicolumn{9}{|c|}{ EBV-positive } \\
\hline 1 & 6.5 & 2.0 & 3.2 & 3 & + & + & - & - \\
\hline 2 & 4.5 & 1.9 & 2.4 & 3 & + & + & - & - \\
\hline 3 & 6.8 & 4.3 & 1.6 & nd & + & nd & nd & nd \\
\hline 4 & 9.4 & 2.8 & 3.3 & 3 & + & + & - & - \\
\hline 5 & 6.2 & 10.7 & 0.6 & 2 & + & + & - & - \\
\hline 6 & 11.1 & 3.3 & 3.3 & 3 & + & + & - & - \\
\hline 7 & 6.3 & 5.7 & 1.1 & 3 & + & + & - & - \\
\hline 8 & 30.3 & 40.4 & 0.7 & 3 & + & + & - & - \\
\hline 9 & 5.8 & 10.8 & 0.5 & 3 & + & + & - & - \\
\hline 10 & 5.7 & 11.2 & 0.5 & 3 & + & + & - & - \\
\hline \multicolumn{9}{|c|}{ EBV-negative } \\
\hline 11 & 5.1 & 8.1 & 0.6 & 1 & - & + & + & + \\
\hline 12 & 2.8 & 15.9 & 0.2 & 1 & - & + & - & - \\
\hline 13 & 4.3 & 11.4 & 0.4 & 1 & - & + & - & - \\
\hline 14 & 1.8 & 12.0 & 0.2 & 1 & - & + & - & - \\
\hline 15 & 6.2 & 7.4 & 0.8 & 1 & - & + & - & - \\
\hline 16 & 4.2 & 15.4 & 0.3 & 0 & - & + & + & + \\
\hline 17 & 3.0 & 12.4 & 0.2 & 1 & - & + & - & - \\
\hline
\end{tabular}

FOXP3 and GrB are expressed as positive cells/HRS cells.

GrB, granzyme B; RIG-I, retinoic acid-inducible gene-I; IRF3, interferon regulatory factor 3; pIRF3, phosphorylated IRF3; INF- $\beta$, interferon- $\beta$; EBV, Epstein-Barr virus; nd, not done

-negative CHLs (Fig. 3, Tables 2 \& 3). Small lymphocytes were also positive for IRF3. The nuclear positivity of pIRF3 was not found in EBV-positive CHL (0/9, 0\%), whereas it was detected in 2 out of 7 cases (29\%) of EBV-negative CHL (Table 3). The expression of IFN- $\beta$ was absent in all cases of EBV-positive CHL (Fig. 3) ; in EBV-negative CHL, IFN- $\beta$ was expressed in the cases in which nuclear translocation of pIRF3 was detected (Fig. 3).

\section{DISCUSSION}

The current study on lesions of CHL showed an increase in $\mathrm{FOXP}^{+}$cells and a decrease in $\mathrm{GrB}^{+}$cells in EBVpositive $\mathrm{CHL}$ of the elderly. In previous studies, when subjects were included from all age groups, $\mathrm{FOXP} 3^{+}$regulatory $\mathrm{T}$ cells (Tregs) dominated the TME of EBV-positive CHL, as shown in the current study. ${ }^{13-15}$ However, in pediatric patients younger than 10 years, there was no difference in the proportion of Tregs between EBV-positive and -negative cases, while $\mathrm{GrB}^{+} \mathrm{CTL}$ was increased in EBV-positive CHL. ${ }^{16}$ These studies indicate the importance of age in the involvement of EBV in CHL. However, the topographic correlation between EBV-positive HRS cells and surrounding cells has not been clear. ${ }^{13-16}$ The current study is the first to show the significance of EBV infection in the modulation of TME in $\mathrm{CHL}$ of the elderly. Our results of quantification of $\mathrm{FOXP}^{+}$ cells and $\mathrm{GrB}^{+}$cells per HRS cell on triple immunostained sections may reflect a close interaction between EBV-
Table 3. Expression of retinoic acid-inducible gene-I (RIG-I), phosphorylated interferon regulatory factor 3 (pIRF3), interferon (IFN)- $\beta$ and CCL20 in Hodgkin and Reed-Sternberg cells

\begin{tabular}{|c|c|c|c|}
\hline & $\begin{array}{c}\text { EBV-positive } \\
(\mathrm{N}=9)\end{array}$ & $\begin{array}{c}\text { EBV-negative } \\
(\mathrm{N}=7)\end{array}$ & Chi-square \\
\hline \multicolumn{4}{|l|}{ CCL20 } \\
\hline 0 & 0 & 1 & $\mathrm{p}<0.01$ \\
\hline $1+$ & 0 & 6 & \\
\hline $2+$ & 1 & 0 & \\
\hline $3+$ & 8 & 0 & \\
\hline \multicolumn{4}{|l|}{ RIG-I } \\
\hline- & 9 & 0 & $\mathrm{p}<0.01$ \\
\hline+ & 0 & 7 & \\
\hline \multicolumn{4}{|l|}{ IRF3 } \\
\hline- & 0 & 0 & ns \\
\hline+ & 9 & 7 & \\
\hline \multicolumn{4}{|l|}{ pIRF3 } \\
\hline- & 9 & 5 & ns \\
\hline+ & 0 & 2 & \\
\hline \multicolumn{4}{|l|}{ IFN- $\beta$} \\
\hline- & 9 & 5 & ns \\
\hline+ & 0 & 2 & \\
\hline
\end{tabular}

EBV, Epstein-Barr virus ; ns, not significant

positive HRS cells and surrounding inflammatory cells.

The constitutive activation of NF- $\varkappa \mathrm{B}$ in HRS cells plays an important role in the pathogenesis of CHL. ${ }^{4}$ The activation of NF- $\varkappa \mathrm{B}$ is critical for the survival of HRS cells, and it also induces the expression of immunosuppressive cytokines, 
TME in classical Hodgkin lymphoma

\section{EBV-positive EBV-negative}
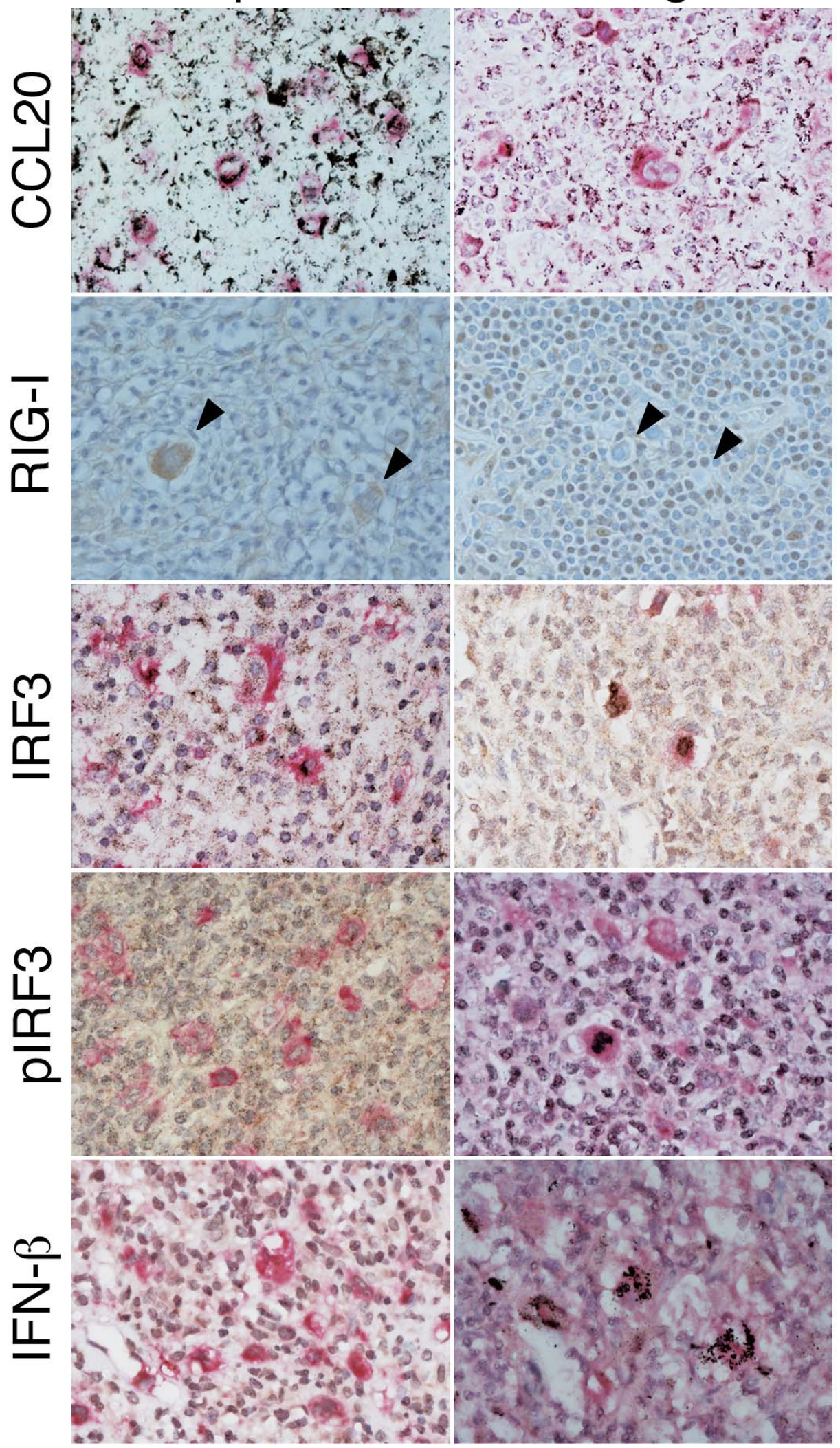

Fig. 3. CCL20 expression and retinoic acid-inducible gene-I (RIG-I) signaling in Epstein-Barr virus (EBV)-positive classical Hodgkin lymphoma (CHL) (case 7) and EBV-negative CHL (case 16). RIGI expression was visualized using diaminobenzidine (as brown), and the HRS cells are indicated with arrowheads. The expression was up-regulated in EBVpositive CHL. CCL20, interferon regulatory factor 3 (IRF3), phosphorylated IRF3 (pIRF3) and interferon- $\beta$ (IFN) are shown in black, and Hodgkin and ReedSternberg cells were stained with CD30 (red). Phosphorylation and nuclear translocation of pIRF3 were found in HRS cells of EBV-negative CHL. 


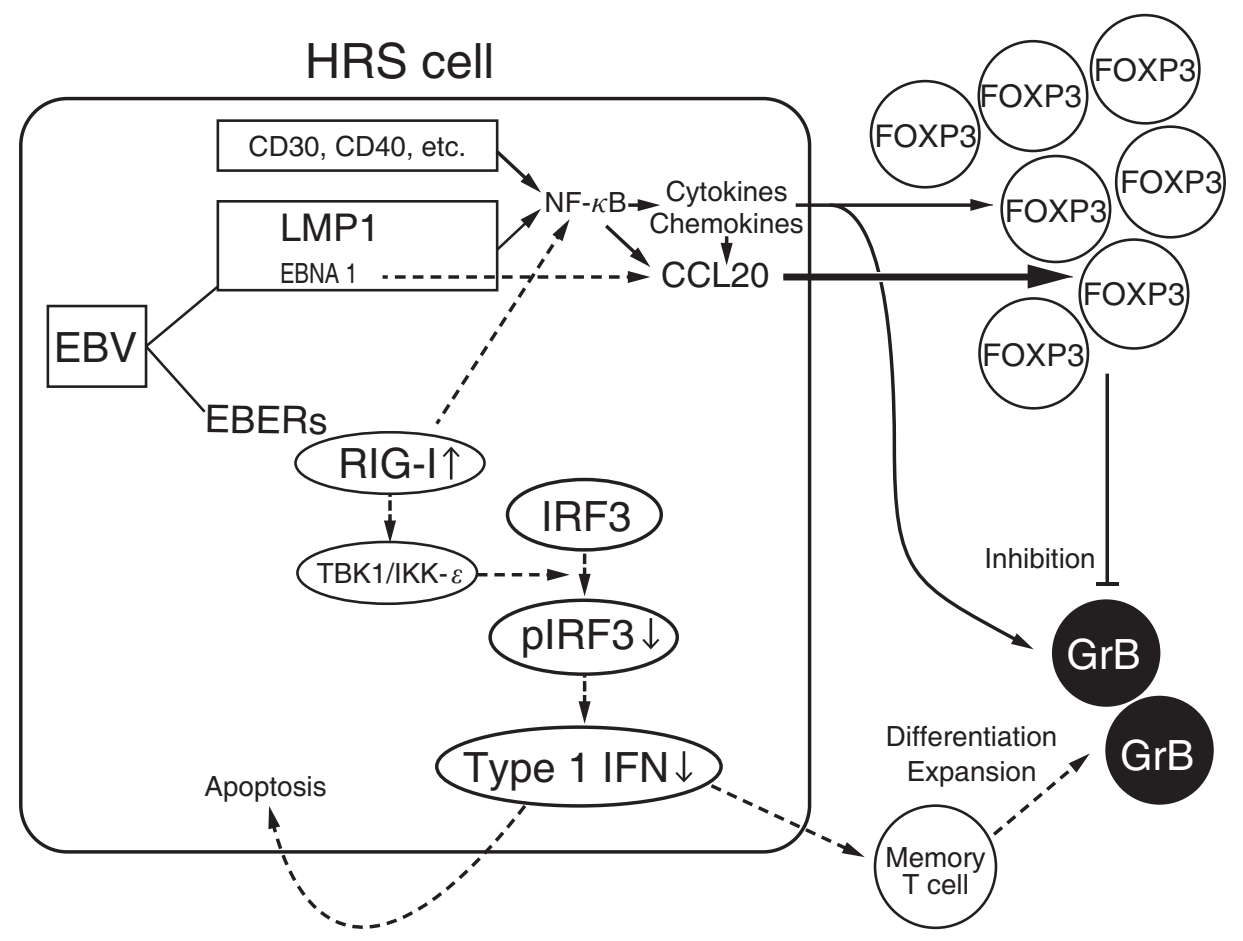

Fig. 4. Intracellular molecular alterations and their effects on cells in the tumor microenvironment.

chemokines and growth factors for the cellular interactions and formation of TME suitable for the survival of HRS cells (Fig. 4). ${ }^{17}$ In EBV-positive CHL, the expression of interleukin-10, an immunosuppressive cytokine, is increased, ${ }^{18}$ and LMP1 may directly induce the migration of Tregs. ${ }^{13}$ The current study suggests the additional mechanisms by which HRS cells interact with TME in EBVpositive CHL.

EBNA1 is an essential factor for the replication of EBV, and it is expressed under the latent infection of EBV in CHL. ${ }^{8}$ It was shown that EBNA1 induces the expression of CCL20 and recruits Tregs in vitro; however, the association of CCL20 expression with Treg infiltration in the primary tumors of CHL was not demonstrated in vivo. ${ }^{14}$ In this study, we confirmed the enhanced expression of CCL20 in EBVpositive HRS cells and its association with infiltration of $\mathrm{FOXP}^{+}$cells. Hence, it is likely that infiltrating $\mathrm{FOXP}^{+}$ cells further inhibit the migration and differentiation of $\mathrm{GrB}^{+}$ cells in TME (Fig. 4). ${ }^{19}$

The current study unveiled the up-regulation of RIG-I in EBV-positive HRS cells. This result is consistent with the up-regulation of RIG-I (DDX58) mRNA in EBV-positive CHL using microarray in a previous study (Ref. 20, in supplementary data). ${ }^{20}$ RIG-I is a pattern recognition receptor against double-stranded RNA, and its interaction with EBER was shown in an EBV-positive BL cell line. ${ }^{10,11}$ The interac- tion activates NF- $x \mathrm{~B}$ and IRF3 and up-regulates the type I IFN in BL cells (Fig. 4). ${ }^{10}$ Since it was shown that NF- $\varkappa$ B is constitutively activated in EBV-positive $\mathrm{CHL},{ }^{4}$ activation of RIG-I signaling may in part contribute to $\mathrm{NF}-\varkappa \mathrm{B}$ activation (Fig. 4). In this study, however, we could find neither phosphorylation of IRF3 nor induction of IFN- $\beta$ in EBV-positive HRS cells, in spite of RIG-I up-regulation. The defect in type I IFN induction in EBV-positive HRS cells may interfere with the expansion and differentiation of memory T cells in TME, leading to the immune evasion of EBV-infected HRS cells (Fig. 4). ${ }^{19}$

The mechanism by which the post-RIG-I signal in EBVpositive HRS cells was down-regulated remains unclear. The difference from the BL cell line may be accounted for by the difference in latency of EBV infection between BL and CHL. ${ }^{10,11}$ In addition, the intracellular signaling may be different between in vitro and in vivo conditions, in the latter of which signals are influenced by neighboring inflammatory cells. $^{10,11}$ The post-RIG-I signaling may also be interfered with by molecules encoded by EBV. It was shown that the immediate-early gene BRLF1 reduced the expression levels of IRF3 and IRF $7^{21}$ and that virion-associated kinase BGLF4 suppressed the transcription of IFN- $\beta$ through the aberrant phosphorylation of IRF3. ${ }^{22}$ These considerations are mostly speculative, and further investigation is required to elucidate the inhibitory mechanism of post-RIG-I signaling in EBV- 
positive CHL.

Our results may provide new insight into the therapeutic strategy for CHL. Although CHL is basically curable, complete eradication of neoplastic cells is difficult, and treatment failure is not uncommon in EBV-positive CHL of the elderly. ${ }^{6}$ Currently, clinical trials of antiproliferative and proapoptotic agents, which target $\mathrm{NF}-\varkappa \mathrm{B}, \mathrm{Akt} / \mathrm{mTOR}$ and bcl2 in HRS cells, are in progress. ${ }^{23} \mathrm{EBV}$-associated molecules or their downstream molecules could be a novel therapeutic target for EBV-positive CHL. Inhibition of CCL20 by targeting EBNA1 may be expected to reduce Treg infiltration. The activation of IRF3 could up-regulate the expression of type I IFN, which in turn induces expansion and differentiation of memory $\mathrm{T}$ cells against EBV-infected HRS cells. Induction of apoptotic cell death of HRS cells could also be anticipated. Consequently, the decrease in the dose of chemotherapeutic agents and the reduction of adverse reactions such as severe immunosuppression or the occurrence of infectious diseases can be achieved.

\section{ACKNOWLEDGMENT}

This study was supported by Grant-in-Aid for Scientific Research (C) from the Ministry of Education, Science, Culture, Sports, Science and Technology, Japan (No. 24591378), to Ryuichi Wada.

\section{REFERENCES}

1 Küppers R: The biology of Hodgkin's lymphoma. Nat Rev Cancer 9:15-27, 2009

2 Eberle FC, Mani H, Jaffe ES: Histopathology of Hodgkin's lymphoma. Cancer J 15:129-137, 2009

3 Aldinucci D, Gloghini A, Pinto A, De Filippi R, Carbone A: The classical Hodgkin's lymphoma microenvironment and its role in promoting tumour growth and immune escape. J Pathol 221:248263, 2010

4 Farrell K, Jarrett RF: The molecular pathogenesis of Hodgkin lymphoma. Histopathology 58:15-25, 2011

5 Jarrett RF, Stark GL, White J, Angus B, Alexander FE, et al.: Impact of tumor Epstein-Barr virus status on presenting features and outcome in age-defined subgroups of patients with classic Hodgkin lymphoma: a population-based study. Blood 106:24442451, 2005

6 Diepstra A, van Imhoff GW, Schaapveld M, Karim-Kos H, van den Berg A, et al:: Latent Epstein-Barr virus infection of tumor cells in classical Hodgkin's lymphoma predicts adverse outcome in older adult patients. J Clin Oncol 27:3815-3821, 2009

7 Keegan TH, Glaser SL, Clarke CA, Gulley ML, Craig FE, et al:: Epstein-Barr virus as a marker of survival after Hodgkin's lymphoma: a population-based study. J Clin Oncol 23:7604-7613, 2005

8 Young LS, Murray PG: Epstein-Barr virus and oncogenesis: from latent genes to tumours. Oncogene 22:5108-5121, 2003

9 Rehwinkel J, Reis e Sousa C: RIGorous detection: exposing virus through RNA sensing. Science 327:284-286, 2010

10 Samanta M, Iwakiri D, Kanda T, Imaizumi T, Takada K: EB virus-encoded RNAs are recognized by RIG-I and activate signaling to induce type I IFN. EMBO J 25:4207-4214, 2006

11 Samanta M, Iwakiri D, Takada K: Epstein-Barr virus-encoded small RNA induces IL-10 through RIG-I-mediated IRF-3 signaling. Oncogene 27:4150-4160, 2008

12 Hodgkin lymphoma. In: Swerdlow SH, Campo E, Harris NL, Jaffe ES, Pileri SA, et al. (eds): World Health Organization Classification of Tumours, WHO Classification of Tumours of Haematopoietic and Lymphoid Tissues. 4th ed, Lyon, International Agency for Research on Cancer (IARC), pp.321334, 2008

13 Marshall NA, Culligan DJ, Tighe J, Johnston PW, Barker RN, et al.: The relationships between Epstein-Barr virus latent membrane protein 1 and regulatory T cells in Hodgkin's lymphoma. Exp Hematol 35:596-604, 2007

14 Baumforth KR, Birgersdotter A, Reynolds GM, Wei W, Kapatai $\mathrm{G}$, et al.: Expression of the Epstein-Barr virus-encoded EpsteinBarr virus nuclear antigen 1 in Hodgkin's lymphoma cells mediates up-regulation of CCL20 and the migration of regulatory $\mathrm{T}$ cells. Am J Pathol 173:195-204, 2008

15 Assis MC, Campos AH, Oliveira JS, Soares FA, Silva JM, et al.: Increased expression of $\mathrm{CD}^{+} \mathrm{CD} 25^{+} \mathrm{FOXP}^{+}$regulatory $\mathrm{T}$ cells correlates with Epstein-Barr virus and has no impact on survival in patients with classical Hodgkin lymphoma in Brazil. Med Oncol 29:3614-3619, 2012

16 Barros MH, Vera-Lozada G, Soares FA, Niedobitek G, Hassan R: Tumor microenvironment composition in pediatric classical Hodgkin lymphoma is modulated by age and Epstein-Barr virus infection. Int J Cancer 131:1142-1152, 2012

17 Steidl C, Connors JM, Gascoyne RD: Molecular pathogenesis of Hodgkin's lymphoma: increasing evidence of the importance of the microenvironment. J Clin Oncol 29:1812-1826, 2011

18 Herbst H, Foss HD, Samol J, Araujo I, Klotzbach H, et al.: Frequent expression of interleukin-10 by Epstein-Barr virusharboring tumor cells of Hodgkin's disease. Blood 87:2918-2929, 1996

19 Welsh RM, Bahl K, Marshall HD, Urban SL: Type 1 interferons and antiviral CD8 T-cell responses. PLoS Pathog 8:e1002352, 2012

20 Chetaille B, Bertucci F, Finetti P, Esterni B, Stamatoullas A, et al.: Molecular profiling of classical Hodgkin lymphoma tissues uncovers variations in the tumor microenvironment and correlations with EBV infection and outcome. Blood 113:2765-3775, 2009

21 Bentz GL, Liu R, Hahn AM, Shackelford J, Pagano JS: EpsteinBarr virus BRLF1 inhibits transcription of IRF3 and IRF7 and suppresses induction of interferon- $\beta$. Virology 402:121-128, 2010

22 Wang JT, Doong SL, Teng SC, Lee CP, Tsai CH, et al.: EpsteinBarr virus BGLF4 kinase suppresses the interferon regulatory factor 3 signaling pathway. J Virol 83:1856-1869, 2009 
Satoh T, et al.

23 Diefenbach C, Steidl C: New strategies in Hodgkin lymphoma: better risk profiling and novel treatments. Clin Cancer Res 19:2797-2803, 2013 